\title{
The Effects of Magnetic Targeted Nano-particles upon Levels of Cytokines in Serum and Synovial Fluid of Experimental Arthritis Rats
}

\author{
Cheng Meng ${ }^{1, a}$, Zhang Dong ${ }^{2}$, Cai Yun ${ }^{1}$ \\ ${ }^{1}$ Department of Rheumatology, Affliated Hospital of Hebei University, Baoding, 071000, P. R. China. \\ ${ }^{2}$ Central Institute for Correctional Police, Baoding, 071000, P. R. China. \\ a277686586@qq.com
}

Keywords: Rheumatoid arthritis; Magnetic targeted Triptolide Nano-particles; Cytokine

\begin{abstract}
Objective: To detect the anti-inflammatory action of magnetic targeted triptolide nano-particles on adjuvant arthritis (AA) rats and the levels of interleukin-6 (IL-6), tumor necrosis factor $\alpha(\mathrm{TNF} \alpha)$ and interleu-kin-10 (IL-10) in serum and synovial fluid, in order to investigate the possible therapeutic mechanism of magnetic targeted triptolide nano-particles on AA. Method: SD arthritis rats were used to make AA models by Fueund's complete adjuvant (FCA). A total of all model rats were randomly divided into two groups, AA control group and triptolide nano-particles group.

Triptolide nano-particles group was administrated with triptolide nano-particles at $40 \mu \mathrm{g} / \mathrm{kg}$ body weight intramuscularly every three days. The rats were sacrificed on the 31st day after the triptolide nano-particles administration. The production of TNF $\alpha$, IL-6 and IL-10 in serum and synovial fluid were detected by enzyme-linked immunosorbent assay (ELISA). Results: Compared with normal control group, the levels of TNF $\alpha$ and IL-6 in serum and synovial fluid of rats in AA control group increased, but the level of IL-10 decreased $(\mathrm{P}<0.01)$. After the treatment of triptolide nano-particles administration, the levels of TNF $\alpha$ and IL-6 in serum and synovial fluid decreased while the level of IL-10 increased. There were significant differences in the cytokines changes between the triptolide nano-particles group and the AA control group $(\mathrm{P}<0.01)$. Conclusion: This study demonstrates that magnetic targeted triptolide nano-particles can possibly modulate the production of TNF $\alpha$, IL-6 and IL-10, which may be one of the mechanisms that triptolide treats the AA and rheumatoid arthritis.
\end{abstract}

\section{Introduction}

Rheumatoid arthritis (RA) is a kind of multiple system inflammatory autoimmune disease which mainly involves peripheral joints. It is performed clinically that the affected joints are arthralgia and arthrocele accompanied by declined functions meanwhile. And the progress of its pathological changes is continuous and repeated. As triptolide is anti-inflammatory and immunosuppressive, it has been applied to treat multiple autoimmune diseases such as RA, which has led to prominent results currently. Magnetic targeted triptolide nano-particles developed in our research group is a kind of new drug delivery system. It can be used in AA rat models to detect the levels change of TNF $\alpha$, IL-6 and IL-10 in serum and synovial fluid and its effects on these cytokines, in order to investigate the therapeutic mechanism that triptolide treats rheumatoid arthritis.

\section{Materials and Methods}

Experimental animals. Clean and health male SD rats (130-150g, provided by Hebei Experimental Animals Research Center). The rats are feed under normal conditions before experiments and during observation period.

Experimental reagent. Triptolide ( provided by Hebei Academy of Medical Sciences, with the purity of 99.7\%); Fueund's complete adjuvant, CFA, Sigma; IL-6, IL-10, TNF $\alpha$ ELISA kit bought from BioSource International; P-Hydroxyazobenzene, IPTS bought from Aldrichimica; ammonia, hydrochloric acid, DMF, TEOS, 6 Ferric chloride hexahydrate, Sodium acetate anhydrous, ethylene 
glycol, n ethane, chloroform, 44 dihydroxybenzaldehyde, CTAB, methylbenzene and ethyl alcohol bought from Beijing Chemical Works.

Model Replication and Animal grouping. 10 of all 50 rats were randomly selected into normal control group, and the rest were used to replicate models. $0.1 \mathrm{~mL}$ CFA was injected subcutaneously to the 40 rats at the pad of right hind foots. Then CFA was injected subcutaneously at the selected five parts between the rats' back and tail head. And $0.05 \mathrm{ml}$ CFA was injected respectively to the five parts. After 15 days, $0.05 \mathrm{ml}$ CFA was injected subcutaneously at the other two newly selected parts. While as regards the normal control group, $9.0 \mathrm{~g} / \mathrm{L}$ sodium chloride injection was injected in the same way. After 30 days since the primary immunization, arthritis index scoring criteria [1] was consulted to evaluate the effects of model replication. The rats that were gave a mark over 6 would be used to carry on the experiment (about 50\%). Then the rats which have been successfully replicated would be divided into two groups: AA control group and triptolide nano-particles group. Each group consists of 10 rats respectively. Then, Literature [2] could be referred to prepare targeting material PDP-MCM-41@SiO2@Fe3O4 which was used for magnetic targeted triptolide nano-particles. Magnetic targeted triptolide nano-particles suspension was produced in high-pressure homogenization. $0.1 \mathrm{~g}$ targeting material was poured into $5 * 10-6 \mathrm{~mol} / \mathrm{L}$ suspension which would be then stirred under blue rays of 450 nanometer wave lengths for two days, in order to prompt targeting material heavy azobenzene structure (cis-trans isomerism) to create powering movement. Consequently the filling of magnetic targeted triptolide nano-particles was completed. After filling, the solid sample could be collected centrifugally through centrifugal machine and rinsed constantly by large quantities of ethyl alcohol until the liquid has been colorless. Then the solid sample with $100 \mathrm{ml} / \mathrm{L}$ propylene glycol was confected into injection which involved $200 \mu \mathrm{g} / \mathrm{ml}$ solid sample. The normal control group, AA control group and triptolide nano-particles group were respectively injected at $9.0 \mathrm{~g} / \mathrm{L}$ sodium chloride injection, $100 \mathrm{ml} / \mathrm{L}$ propylene glycol and triptolide nano-particles injection. The dose for the three groups was equally $0.4 \mathrm{ml} / \mathrm{kg}$ delivered by intramuscular injection at hind leg every 3 days. Then the three groups would be introduced to the given inflammation by external magnetic field for the purpose that the release of effective drug triptolide nano-particles could be finished under the light irradiation of 540 nanometer wave lengths.

Sampling. The experimental rats should be weighed and received abdominal anaesthesia by $2.5 \mathrm{ml} / \mathrm{kg}$ pentobarbital sodium $(20 \mathrm{ml} / \mathrm{L})$. And the blood was sampled from aorta abdominalis and the serum was isolated in a conventional way in order to detect cytokines; the rats were fixed upward and then knee joints were unfolded. After that, $1 \mathrm{ml}$ sodium chloride injection $(9.0 \mathrm{~g} / \mathrm{L})$ was used to wash articular cavity and sample joint cavity fluid in order to detect cytokines.

The Detection of Cytokines. To operate on the basis of the introduction offered by ELISA kit.

Statistics Method. SPSS 11.5 was used to conduct analysis. The differences existed in groups could be tested by t detection and the results were shown in " $\bar{x} \pm s$ ”.

\section{Results}

The Level of TNF $\alpha$ in Serum and Synovial Fluid. In AA control group, the level of TNF $\alpha$ in serum and synovial fluid was the highest. After treatment, the level of TNF $\alpha$ in serum and synovial fluid decreased obviously, which proved that the effective constituents in magnetic targeted triptolide nano-particles could reduce the level of TNF $\alpha(\mathrm{P}<0.01)$. And compared with the normal control group, there was no significant difference in the cytokines changes between the triptolide nano-particles group and the AA control group ( $P>0.05)$, as is shown in Table 1 .

The Level of IL-6 in Serum and Synovial Fluid. In AA control group, the level of IL-6 in serum and synovial fluid was the highest. After treatment, the level of IL-6 in serum and synovial fluid decreased obviously, which proved that the effective constituents in magnetic targeted triptolide nano-particles could reduce the level of IL-6 $(\mathrm{P}<0.01)$. However, the level of IL-6 failed to reach that of the normal rats $(\mathrm{P}<0.05)$, as is shown in Table 1 .

The Level of IL-10 in Serum and Synovial Fluid. In AA control group, the level of IL-10 in serum and synovial fluid decreased obviously. After treatment, the level of IL-10 has increased 
apparently, which proved that the effective constituents in magnetic targeted triptolide nano-particles could raise the level of IL-10(P<0.01). And compared with the normal control group, there was no significant difference in the cytokines changes between the triptolide nano-particles group and the AA control group ( $\mathrm{P}>0.05)$, as is shown in Table 1.

\begin{tabular}{|c|c|c|c|c|c|c|c|}
\hline \multicolumn{8}{|c|}{ Table1 The Levels of TNF $\alpha$, IL-6, IL-10 in Serum and Synovial Fluid $(\mu \mathrm{g} / \mathrm{ml}, \mathrm{n}=10, \bar{X} \pm S$ ) } \\
\hline \multirow[b]{2}{*}{ Groups } & \multirow[b]{2}{*}{$\mathrm{n}$} & \multicolumn{2}{|c|}{$\mathrm{TNF} \alpha$} & \multicolumn{2}{|c|}{ IL-6 } & \multicolumn{2}{|c|}{ IL-10 } \\
\hline & & Serum & $\begin{array}{c}\text { Synovial } \\
\text { Fluid }\end{array}$ & Serum & Synovial Fluid & Serum & $\begin{array}{c}\text { Synovial } \\
\text { Fluid }\end{array}$ \\
\hline $\begin{array}{l}\text { The } \\
\text { Normal } \\
\text { Control } \\
\text { Group }\end{array}$ & 10 & $\begin{array}{c}13.05 \pm 3.8 \\
7\end{array}$ & $18.41 \pm 4.21$ & $23.65 \pm 3.35$ & $19.38 \pm 2.21$ & $23.47 \pm 2.65$ & $15.68 \pm 2.53$ \\
\hline $\begin{array}{l}\text { AA } \\
\text { Control } \\
\text { Group }\end{array}$ & 10 & $\begin{array}{c}36.12 \pm 8.8 \\
1^{\star \star}\end{array}$ & $\underset{\star}{44.16 \pm 8.93^{\star}}$ & $76.54 \pm 6.76^{\star \star}$ & $\underset{\star}{88.69 \pm 10.53^{\star}}$ & $17.52 \pm 2.05^{\star}$ & $\underset{\star}{10.59 \pm 2.94^{\star}}$ \\
\hline $\begin{array}{c}\text { Triptolid } \\
\text { e } \\
\text { Nano-pa } \\
\text { rticles } \\
\text { Group }\end{array}$ & 10 & $\begin{array}{c}15.34 \pm 3.5 \\
4^{\mathbf{4}}\end{array}$ & $22.54 \pm 4.67^{\mathbf{\Lambda}}$ & $\begin{array}{c}42.43 \pm 5.72^{\boldsymbol{\Lambda}} \\
\star\end{array}$ & $\begin{array}{c}48.64 \pm 5.98^{\boldsymbol{\Lambda}} \\
\star\end{array}$ & $21.23 \pm 2.93^{\mathbf{\Lambda}}$ & $14.72 \pm 1.87^{\mathbf{\Lambda}}$ \\
\hline
\end{tabular}

Compared with the normal control group, $\star \mathrm{P}<0.05, \star \star \mathrm{P}<0.01$; Compared with AA control group, $\mathbf{\Delta} \Delta \mathrm{P}<0.01$

\section{Discussions}

TNF $\alpha$ and IL-6 have significant effect on the attack and joints injury of rheumatoid arthritis (RA). TNF $\alpha$ could take part in the attack and development of RA through various ways, such as activating endothelial cells and promoting the synthesis and release of inflammatory factors [3]. Meanwhile IL-6 can enhance the effect of TNF $\alpha$ and IL-1, and as a result is regarded as amplification factor for certain biological effect of TNF $\alpha$. Up to now, researches have shown that the levels of TNF $\alpha$ and IL-6 in the blood and lesions fluid of RA patients have increased significantly, and that the levels of TNF $\alpha$ and IL-6 have obviously decreased with the improvement of simultaneous arthritis disease activity index after treatment. If anti-TNF $\alpha$ monoclonal antibody and anti-IL-6 monoclonal antibody were used to treat RA, synovitis and cartilage destruction in lesion joints would be controlled and the patients' symptoms and signs would be relieved evidently. Moreover, the inflammatory index like MMP-1, MMP-2, and C - reactive protein in serum has also decreased [4, 5]. In this experiment, it can be also observed that TNF $\alpha$ and IL-6 in rats' blood and synovial fluid have distinctly increased, which suggested that they have the equally important effect on AA attack. And after the treatment of magnetic targeted triptolide nano-particles, both of the levels of TNF $\alpha$ and IL-6 in blood and synovial fluid can decline obviously, which proved the fact that triptolide nano-particles may possibly treat RA by reducing the levels of TNF $\alpha$, IL- 6 and other inflammatory cytokines.

The therapeutic effect of IL-10 on arthritic can be realized through multiple mechanisms. First, it can restrain the production of pro-inflammatory cytokines in order to reduce the emigration of lymphocyte to lesion joints; meanwhile, it can increase the production of endogenous anti-inflammatory substances as soluble tumor necrosis factor receptor and IL-1 receptor antagonist, in order to further control IL-1 $\beta$ and TNF $\alpha$ [6]; in addition, IL-10 can also restrain the release of epoxidase-2[7]; it can also control the expression of matrix metalloproteinase and enhance the expression of the inhibitor factors in metalloproteinase for the purpose of protecting chondrocyte[8]. The author has observed in this experiment that the level of IL-10 in serum and synovial fluid of AA control group has decreased. And after treatment, the level of IL-10 recovered to be normal, which suggested that the effective constituents may directly or indirectly increase the level of IL-10 in peripheral blood and lesion joints and then treat AA therapeutically. 
Through regulating the production of cytokines as IL-6, IL-10 and TNF $\alpha$ in serum and synovial fluid, magnetic targeted triptolide nano-particles can realize the infiltration of inflammatory cells, restrain the formation of synovial hyperplasia and pannus and alleviate the destruction of cartilage for the final purpose of the treatment of AA. It is also the part of the mechanisms that triptolide treats rheumatoid arthritis.

\section{References}

[1] Larson P. Homologous type II collagen induced arthritis in rats [J]. ArthritisRheum, 1990, 339 (5): 693 697.

[2] Meng Chen, Dong Zhang et al.On a site-specific delivery system based on core-shell structured magnetic particles: Preparation and characterization [J]. Optical Materials, 36 (2013) 321-327

[3] Lu Yi. Advance in Research on Pathogenesis of Rheumatoid Arthritis [J]. Foreign Medical Sciences(Section of Immunology Foreign Medical Sciences), 2001, 24(5): 256 258

[4] Lipsky PE, vanderHeijde DM, StClair EW,et al.Infliximab and methotrexate in the treatment of rheumatoid arthritis. Anti-TumorNecrosis is Factor Trial in Rheumatoid Arthritiswith Concomitant Therapy Study Group [ J]. N Engl JMed, 2000, 343(22): 1594 1602.

[5] ZHOU Guang-yu, GUO Jia-long, BI Li-qi. Interleukin-6 levels in serum and synovial fluid in patients with arthritis [J]. Journal of Jilin University of (Medicine Edition), 2004, 30(5): 772 774.

[6] Whalen JD, Lechman EL, Carlos CA,et al. Adenoviral transfer of the IL-10 gene periarticularly to mouse pawssuppresses development of collagen-induced arthritis in both injected and uninjected paws [J]. J Immuno,l 1999, 162(6):3625 3632.

[7] Wu Yuanjun, Chen Ronghua \& Chen Jiqing, et al. IL-10 downregulates expression of cyclo-oxygenase-2 and its mediated PGE2 production in human mesangial cells [J]. Immunological Journal, 2001, 17(6): 421 424.

[8] SeitzM, Loetscher P, Dewald B,et al. Interleukin-10 differentially regulates cytokine inhibitor and chemokine release from blood mononuclear cells and fibroblast [J]. Eur JImmuno,1 1995, 25(4): $1129 \sim 1132$. 